\title{
Itinéraires Itinéraires
}

Littérature, textes, cultures

Expérimenter en langues. Traduction, plurilinguisme et intermédialité dans les avantgardes poétiques depuis 1960

Experimenting in Tongues: Translation, Plurilinguism, and Intermediality in Avant-Garde Poetry since 1960

\section{Mathias Kusnierz}

\section{OpenEdition}

Journals

Édition électronique

URL : http://journals.openedition.org/itineraires/3998

DOI : 10.4000/itineraires.3998

ISSN : 2427-920X

Éditeur

Pléiade

Référence électronique

Mathias Kusnierz, «Expérimenter en langues. Traduction, plurilinguisme et intermédialité dans les avant-gardes poétiques depuis $1960 »$, Itinéraires [En ligne], 2017-3 | 2018, mis en ligne le 15 juin 2018, consulté le 01 mai 2019. URL : http://journals.openedition.org/itineraires/3998 ; DOI : 10.4000/ itineraires.3998

Ce document a été généré automatiquement le 1 mai 2019.

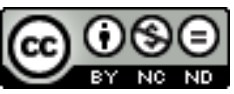

Itinéraires est mis à disposition selon les termes de la licence Creative Commons Attribution - Pas d'Utilisation Commerciale - Pas de Modification 4.0 International. 


\title{
Expérimenter en langues. Traduction, plurilinguisme et intermédialité dans les avant-gardes poétiques depuis 1960
}

\author{
Experimenting in Tongues: Translation, Plurilinguism, and Intermediality in \\ Avant-Garde Poetry since 1960
}

Mathias Kusnierz

Depuis les années 1960 et l'émergence des secondes avant-gardes historiques, le débat sur la production poétique en France a longtemps opposé poésies lyrique et littéraliste. Ce débat continue de susciter de nécessaires prises de position (Gleize 2011: 27-30). Les partisans de la première militent pour un renouvellement du lyrisme qui ne ferait pas table rase de « la plus ancienne, [...], la plus riche composante » (Di Manno 1995 : 11) de la poésie européenne, tandis que les thuriféraires de la seconde affirment la nécessité d'inventer de nouvelles procédures d'écriture permettant de court-circuiter les traditions poétiques passées. Sans nier cette ligne de partage esthétique et politique (Maulpoix $2000: 7-10$ et 41-42; $2002: 233-235)$, je propose plutôt d'envisager l'histoire de la poésie depuis la fin des années 1950 à partir de la question de ses supports. Elle est centrale. Il suffit, pour s'en convaincre, d'observer le foisonnement des différents courants poétiques aujourd'hui : poésie sonore, poésie-performance, poésie concrète ou visuelle, poésie vidéo, poésie numérique et même poésie en langue des signes (Rose 2006) fleurissent à côté de la poésie en livres ou en revues. Je propose de regrouper sous la bannière « poésie élargie " l'ensemble des pratiques poétiques définies par la sortie hors de la page imprimée. Je l'envisagerai conjointement aux avant-gardes dites «textualistes", regroupées à partir des années 1960 autour de revues comme Tel Quel, Change et TXT, puis Manteia et Tartalacrème dans les années 1970 et 1980 et dont le programme a consisté en un renouvellement de l'écriture poétique à partir de la question de la langue, de ses écarts et de ses manipulations possibles. Mon objectif sera de montrer qu'au-delà de la 
ligne de partage entre langue et supports d'inscription, ces différentes pratiques expérimentales poursuivent un même but avant-gardiste.

\section{Naissances simultanées : le Big Bang de la poésie élargie}

2 Il est frappant de constater que la poésie numérique apparaît presque en même temps que l'expression " poésie sonore » mais qu'elle jouit pourtant d'une exposition bien plus faible. Ce constat m'a poussé à me demander si le médium même de la poésie numérique n'était pas l'une des causes de cette réception congrue, et à tenter de comprendre les rapports qu'elle entretient avec les poétiques textualistes. La poésie numérique naît en effet avec les premiers textes stochastiques de Theo Lutz et leur publication dans la revue augenblick (Lutz 1959 et 1960 ; Bense 1969), tandis que le syntagme " poésie exclusivement sonore » apparaît en 1958 dans un texte collectif de la revue -grâmmeS consacré à François Dufrêne. Chacun à leur manière, ces deux événements marquent la sortie du poème hors du champ de la page imprimée. Si, pour reprendre l'expression de Marshall McLuhan (1962 : 271-279), la « galaxie Gutenberg » correspond à un état primitif du développement de la poésie, alors la poésie élargie et ses multiples supports correspondent à une forme de Big Bang. Il serait logique de penser que, dès cet instant, la poésie abandonne la question de la langue et de l'écriture au profit d'une approche rigoureusement matérialiste de son inscription et de son apparition, un tournant qu'Apollinaire avait déjà amorcé avec Calligrammes (1918). Mais il n'en va pas ainsi : tandis que la poésie sonore et la poésie numérique se développent, les avant-gardes textualistes continuent d'explorer la question de l'articulation de la langue et de l'écriture, tout en prenant acte des conquêtes de la poésie sonore et de la poésie concrète (Prigent et Gorillot 2009: 88-89, 126-134). L'Main (1975), Power/Powder (1977) et Cuf-glotte (1978) de Christian Prigent, ou La Danse $d u$ dos (1988) de Jacques Demarcq sont caractéristiques d'une interrogation théorique sur la langue et ses écarts qui passe par l'arsenal formel et typographique de la poésie concrète. $\mathrm{Au} \mathrm{xx}^{\mathrm{e}}$ siècle, l'histoire de la poésie se scinde donc en deux voies parallèles, qui évoluent conjointement mais qu'il y a apparemment lieu d'opposer : l'une continue d'explorer l'écriture, la langue et la page imprimée (poésie visuelle, poésie concrète, lettrisme, avant-gardes textualistes, etc.), l'autre cherche à se redéfinir en poésie élargie et explore d'autres supports (poésie sonore, poésie-performance, poésie numérique). Ces deux voies sont également expérimentales et se revendiquent comme telles (Prigent 2004 : 85-88, 102 ; Donguy 1985 : 74 ; $2007: 7$ ).

3 La poésie élargie correspond à un tel changement de paradigme. Je souhaite montrer ici que, dans les pratiques contemporaines de la poésie numérique, de la poésie concrète ou de la poésie sonore, une même attention à l'étranger et aux langues étrangères se manifeste. Expérimenter d'autres supports pour la poésie, c'est encore expérimenter en langues, de sorte que l'expérimentation avec le médium rejoue, en des termes renouvelés, l'affranchissement face aux idéologies qui constitue le projet avant-gardiste. Je voudrais donc également faire apparaître ici les continuités secrètes qui relient la poésie élargie aux poétiques textualistes et montrer la cohérence profonde des différents projets avantgardistes et expérimentaux, par-delà leur diversité, de façon à exposer la manière dont la littérature postmoderne prolonge et amplifie le projet poétique moderne plutôt qu'elle n'y met fin. 
Pour le dire plus brièvement, mon hypothèse est la suivante : dans la poésie élargie, c'est le médium qui a repris à son compte les interrogations sur le langage. Ces interrogations se sont en effet déplacées du texte vers le médium et, dans cette migration, se sont disséminées puis incarnées en une multitude d'enjeux et de "sites" (son, vidéo, performance, etc.) qui correspondent aux différentes pratiques poétiques d'aujourd'hui.

\section{Retour sur les avant-gardes textualistes}

Les avant-gardes textualistes des années 1960 se caractérisent par la volonté de tenir compte, dans l'écriture poétique, de la pluralité des langues et des langages. Sous l'influence de grands modèles plurilingues comme Ezra Pound, James Joyce, Ernst Jandl ou Oskar Pastior, des écrivains comme Denis Roche, Christian Prigent ou Jean-Pierre Verheggen ont travaillé à élargir le champ linguistique potentiel de leur écriture en y incluant dialectes, jargons, patois ou idiomes, tous étrangers à leur langue maternelle. Dans ces pratiques, la poésie s'est renouvelée par des opérations de traduction internes, proposant au lecteur une expérience de lecture "en langues", que l'on pourrait aussi nommer une expérience de «lecture traductrice », au cours de laquelle il ne cesse d'aller d'une langue à l'autre et de revenir à sa langue d'origine pour mieux la quitter à nouveau. La Liste des langues que je parle ${ }^{1}$ de Christian Prigent (1997 : 28-31) intègre ainsi, dans un texte en langue française, une litanie de noms de langues étrangères ou imaginaires dont la graphie ne ressortit pas au français académique. Il s'agit donc d'un texte écrit en « plus d'une langue » (Cassin 2012), autant qu'un texte qui, au deuxième degré, procède à la fusion d'une infinité d'idiomes dans une langue française malmenée, écartelée et déformée. Les noms de ces idiomes sont, dans le texte, comme la promesse d'espaces linguistiques infinis.

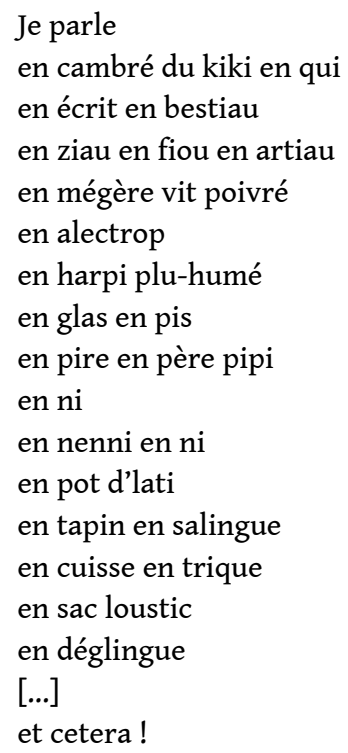

6 La manière dont Prigent lit ce texte fait entendre qu'il est écrit pour être performé en public. L'auteur a d'ailleurs théorisé précisément son usage de la voix et de la lecture publique en évoquant la tension que la lecture à voix haute crée par rapport au texte silencieux (1984: 36). Le fait que ce travail sur la langue passe par le truchement de la performance suggère à nouveau qu'il n'y a pas de frontière nette ni d'incompatibilité entre l'approche textualiste et la poésie élargie. 
Fig. 1. Extraits de Jacques Demarcq, Les Zozios, Caen, Nous, 2008.

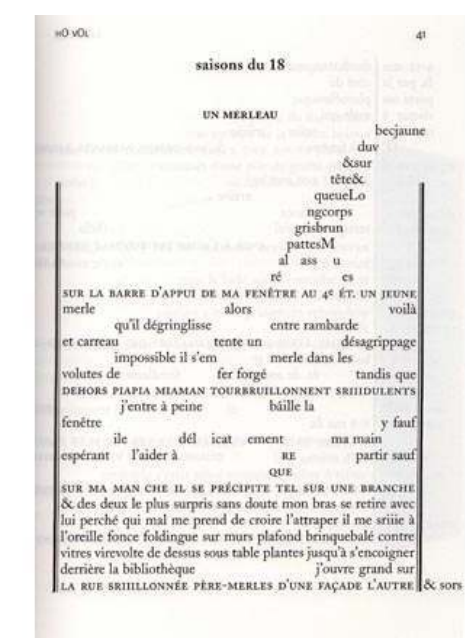

\author{
\& l'aléa les aile à n'importe, improviste \\ Dés jeté \\ risque-trous \\ à dévisser des piste $s$ \\ Ils vont idiots \& cons dans l'air, c ha r p ie de chances \\ Qui bat, rebat les cartes où le $s$ avoir nous fiche
}

Dans Les Zozios (fig. 1), Jacques Demarcq (2008) confronte, dans un même espace textuel, la langue française à toute une panoplie d'écarts linguistiques et de procédés sonores ou visuels qui y restituent le chant des oiseaux. Demarcq et Prigent ne proposent pas des textes qui soient la traduction en langues d'un en-deçà ou d'un au-delà du langage. Ils conçoivent des opérations linguistiques qui complexifient - à l'aide de mécanismes qui relèvent de la traduction - le rapport du locuteur à sa propre langue. Le poème devient alors l'outil qui permet au lecteur de plier sans cesse le langage sur lui-même, c'est-à-dire qu'il creuse une distance toujours plus grande entre le locuteur et sa langue jusqu'à supprimer l'évidence de la parole elle-même. Le texte s'y divise et s'y ramifie en plusieurs idiomes, de même que certains mots se décomposent concrètement en plusieurs solutions de lecture dans Hacettepe University Bulletin (Prigent 1976, fig. 2). Réfléchissant à un problème formel similaire dans la poésie numérique, Philippe Bootz (2006: 379-380) a évoqué les textes informatiques fondés sur des combinatoires et des permutations de signes: c'est là l'indice que certaines procédures d'écriture propres aux avant-gardes textualistes circulent jusque dans la poésie élargie. De la même façon, les cut-ups de Brion Gysin et William Burroughs (1961) ou de Denis Roche (1980) sont une version manuelle des textes stochastiques de Theo Lutz. Mais dans Liste des langues que je parle ou Les Zozios ces mécanismes linguistiques sont intégrés au texte en langue française, de sorte que lire le texte, c'est toujours le lire dans sa propre langue et le lire en "plus d'une langue ». En entrant dans le texte, le locuteur fait l'expérience d'un étoilement de sa faculté linguistique. Et dans cette expérience, la poésie affranchit son lecteur de ses appartenances identitaires, de ses réflexes de pensée et de l'assignation aux idéologies qui lui est faite. 
Fig. 2. Extraits de Christian Prigent, Hacettepe University Bulletin, Nœuds-les-Mines, Alin Anseeuw, 1976.
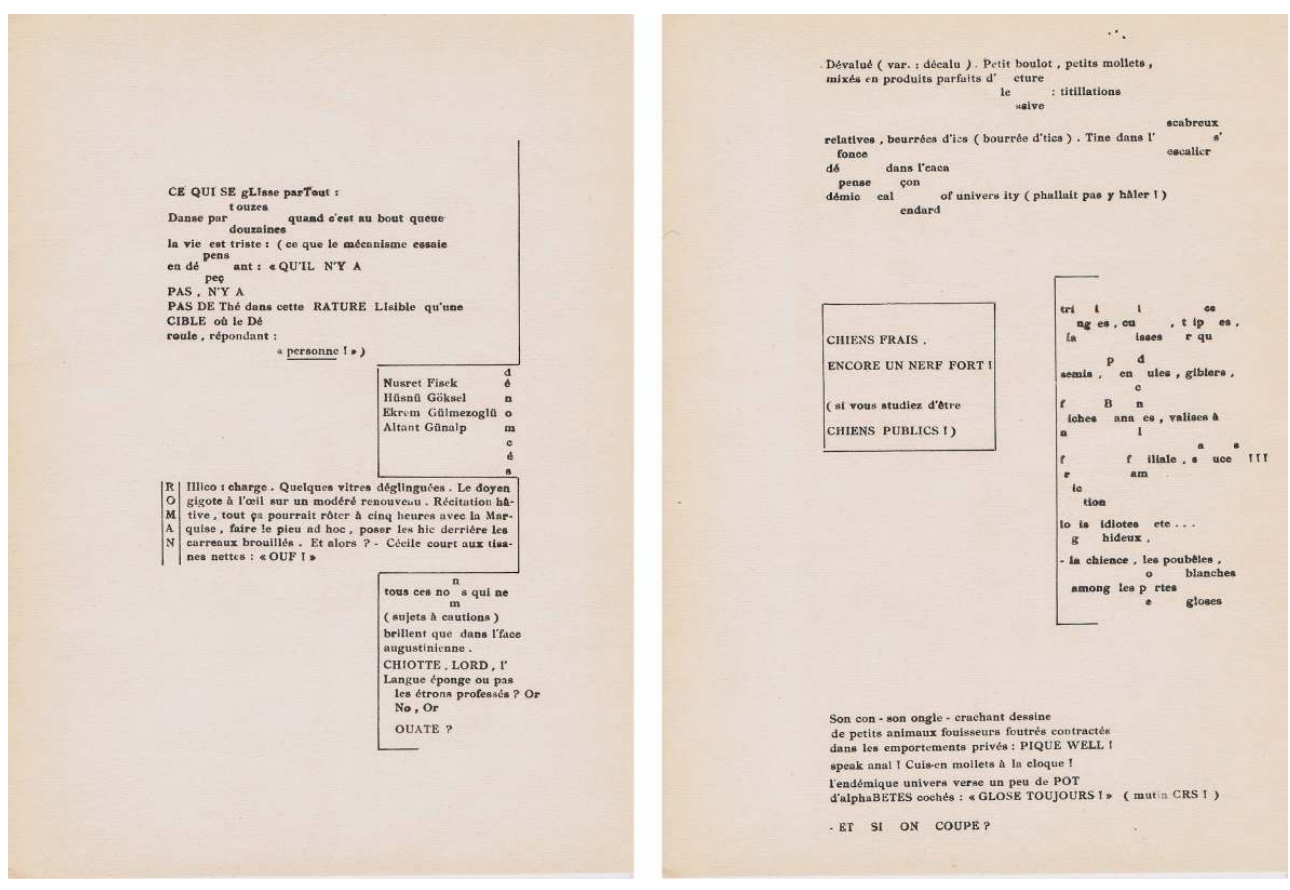

\section{La poésie sonore et la poésie numérique, ou la sortie hors de la page imprimée}

La poésie sonore excède le champ de l'imprimé en proposant des formes qui ne sont ni transcriptibles, ni réductibles à l'écrit, tandis que la poésie numérique procède à une dissémination du poème hors de la page imprimée. En effet, quand bien même le texte en serait simple, le poème sonore n'existe que dans sa projection et dans l'expérience qu'en fait l'auditeur. Bien que le texte en ait été publié, Vaduz de Bernard Heidsieck (2008) ${ }^{2}$ n'existe pas hors de la confrontation, en temps réel, de sa lecture par l'auteur et de la diffusion de la bande magnétique pré-enregistrée, avec tous les effets de décalage, d'écho, de superposition et de réverbération appliqués à la voix d'Heidsieck que cette confrontation implique. Les bruits de foule qui se font entendre à la fin de l'enregistrement témoignent d'ailleurs de ce que le poème ne saurait se réduire ici au seul texte. Il tient ainsi en la juxtaposition d'une lecture et d'un enregistrement. La poésie numérique rejoue cette sortie hors de la page imprimée mais, plus encore, elle procède à une dissémination des supports constitutive des technologies numériques: disquettes, CD-Rom, écrans, hologrammes ou installations sonores, etc. Cette dissémination prive le poème de lieu propre (Stefans 2000 ; Saemmer et Dufrêne 2014). L'expérience que le lecteur fait du poème s'en trouve nécessairement transformée. Qui plus est, la multiplication des formats liés aux nouvelles formes de poésie tend à transcender des catégories - art numérique, vidéo, cinéma expérimental, vidéopoème, poésie numérique, etc. - qui, dès lors, se révèlent extrêmement poreuses les unes aux autres, voire caduques. Comment, par exemple, catégoriser Code Movie 1 de Giselle Beiguelman (2004, fig. 3) ? Il s'agit ici d'art numérique (l'auteure se définit comme "media artist $\left.{ }^{3} »\right)$ autant que de poésie. Si l'on place le travail de Beiguelman en regard des Poems de Carl Andre (2014) ou 
des Konstellationen d'Eugen Gomringer (1995, fig. 4), nos catégories de réception se brouillent un peu plus encore, puisque les travaux d'Andre et de Gomringer seront perçus tout à la fois comme des œuvres plastiques et poétiques.

Fig. 3. Extraits de Giselle Beiguelman, Code Movie 1, 2004.
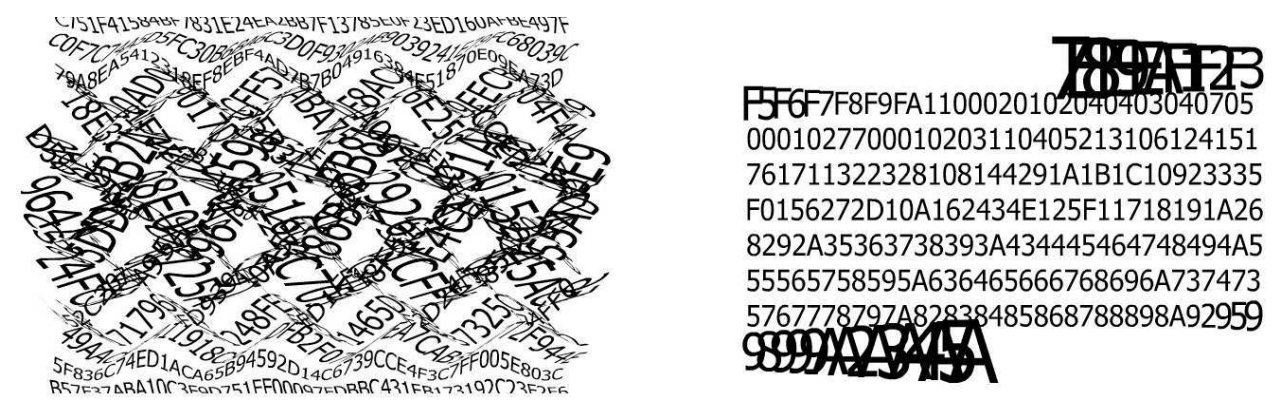

Fig. 4. Extraits de Eugen Gomringer, Konstellationen, Vienne, Splitter, 1995.
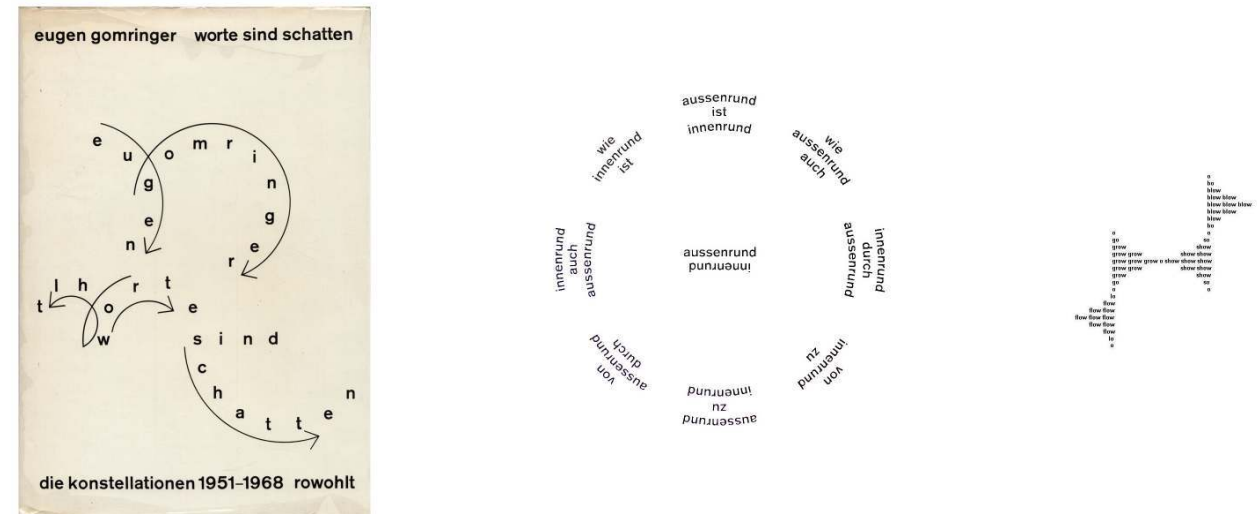

\section{Comment la question des langues rejaillit dans celle des supports}

Ce bref rappel historique et ces quelques exemples me permettent d'affirmer que la poésie sonore et la poésie numérique à sa suite substituent la question du support à celle de la multiplicité des langues et de leur articulation dans l'écriture, enjeux explorés par les avant-gardes textualistes dans les années 1960. Aux outils de la linguistique et de la traductologie que nous utilisons pour rendre compte des pratiques poétiques textualistes, il faudrait donc substituer des outils venus de l'archéologie des médias, des théories de l'intermédialité (Bolter 1991: 65-74; Bolter et Grusin 2000 : 31-44, 52-62, 132-145) et des visual studies. Les réflexions de Lisa Gitelman (2006: 61-64, 95-97) sur la nature transactionnelle des médias fournissent des outils conceptuels qui nous permettent de comprendre en quoi la poésie numérique induit une transformation profonde de l'expérience de lecture. Le poème y est l'espace, l'objet et surtout l'interface d'une transaction, plus qu'un texte qui appelle le lecteur à le déchiffrer. Et puisque bien souvent, il se joue (au plein sens du terme) sur un écran, il constitue une expérience dont la durée est déterminée par le support lui-même. Alexandra Saemmer et Bernadette Dufrêne (2014) expliquent ainsi que la vitesse d'exécution propre à une machine transforme sensiblement l'expérience que le lecteur fait du poème : en cela, comme chez 
Heidsieck, le poème numérique réside dans l'épreuve que l'on en fait plutôt que dans un texte idéalement isolable de ses conditions d'expérience. Par conséquent, le texte n'est pas l'objet d'une lecture mais d'un échange avec le lecteur : celui-ci cède au poème un temps que le dispositif technique transforme en expérience poétique.

Mais peut-on dire pour autant que la poésie numérique s'est totalement écartée de la question des langues ? Je propose de considérer plutôt qu'elle l'a élargie, en en proposant une entente renouvelée par la prise en compte du support du poème. Les pratiques multiples de la poésie numérique exigent, pour que le poème advienne, que l'écrivain conçoive un chiffre ou un code, c'est-à-dire une langue qui s'écarte de la langue courante et de la langue maternelle. En suivant Florian Cramer (2004: 263-276), qui définit la poésie numérique comme un art du code, on imagine sans peine le dispositif qui exposerait leurs pages de code en regard des écrans qui affichent les poèmes. Quant aux pratiques poétiques intermédiales, telles que celle d'Alessandro de Francesco par exemple, elles relèvent indirectement de la traduction. Jakobson (1963: 78-86) avait proposé d'inclure la traduction intersémiotique - qu'il définit comme «l'interprétation des signes linguistiques au moyen de systèmes de signes non linguistiques» $(1963: 79)$ et qui relève, comme telle, de l'intermédialité - dans les trois cas de figure possibles de la traduction. Si l'on suit le théoricien russe, les phénomènes d'intermédialité relèveraient donc, en droit, de la traduction et par conséquent du langage. Mais c'est là, certes, une conception fort large de la traduction. Si l'on considère plus prudemment que la traduction intersémiotique ne relève que métaphoriquement de la traduction, au moins peut-on la comprendre comme une manière de déplacer les procédures formelles linguistiques et traductrices des poétiques textualistes vers d'autres sites que le texte et la page imprimée.

Fig. 5. Extraits de Alessandro de Francesco, Augmented Writing, Rome, La Camera Verde, 2013.
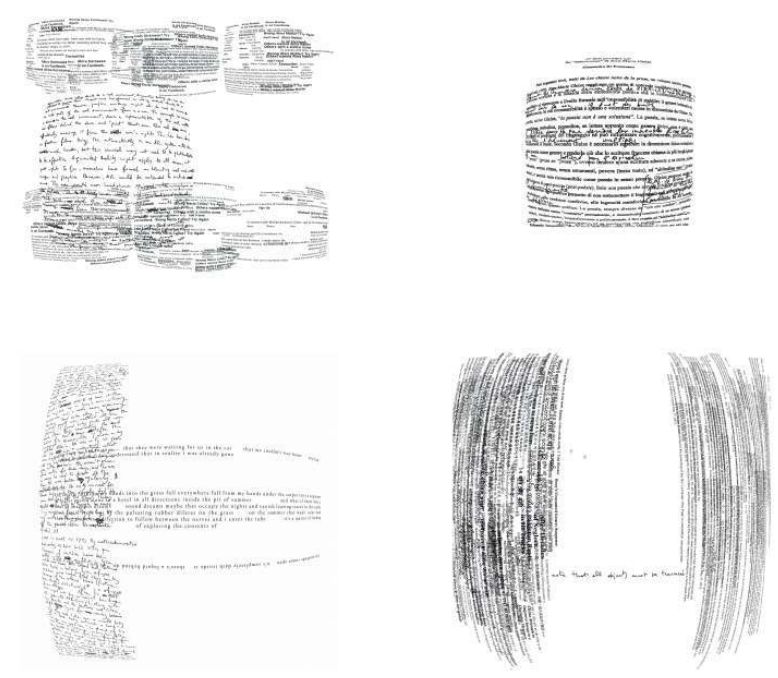
11 Dans les travaux d'Alessandro de Francesco (2016: 9-52, 32-37, 43, 81-85), tels que Corps étranger en mouvement ascensionnel ou La Vision à distance, le poème devient le support d'une performance qui hésite de manière indécidable entre lecture publique, performance artistique et concert de sound design: toutes catégories rendues soudain caduques devant le travail d'indétermination accompli par l'auteur ${ }^{4}$. Le texte se mêle au bruit et s'y dissout, les voix acquièrent une dimension matérialiste qui oblitère ce qu'elles énoncent, comme si s'effaçait ici la frontière entre la langue et le son. Mais, de fait, ce travail interroge la frontière par-delà laquelle le langage cesse d'être langage. Dans d'autres de ses travaux, le poème entretient volontairement une ambiguïté profonde entre le statut de texte et celui d'objet plastique. S'il est possible de les réduire à l'état de pages imprimées manifestant un travail formel sur la circulation des langages préfabriqués et des usages de la communication courante contemporaine, les textes de la série Augmented Writing (2013, fig.5), publiés en volume et tirés sur papier photographique de grand format, exhibent leurs dimensions visuelle, plastique et graphique $^{5}$. Pour fabriquer ces images de textes, de Francesco a recours a plusieurs procédés. Soit le poème fait l'objet d'un traitement numérique qui en déforme l'image, par exemple pour donner l'illusion d'avoir été imprimé sur un support courbe; soit l'objet constitué se révèle un montage de différents textes, superposés jusqu'à saturation de la page ; soit encore le poème se retrouve biffé, raturé ou caviardé et acquiert, par ces opérations, une dimension plastique qui prend le pas sur la dimension textuelle, dans un geste qui renverse, d'une certaine façon, celui qu'accomplissait cy Twombly en intégrant des graffiti parfois illisibles dans ses toiles (Prigent 1996: 31-43). Or, comme chez Twombly, c'est toujours la langue qui est au centre des opérations plastiques, parce que l'image réclame d'être déchiffrée et, en se révélant illisible, fait appel autant qu'elle met en déroute les facultés linguistiques et la culture livresque du regardeur (Barthes 2002 : 688-720). Examinant les poèmes de Susan Howe (1993, fig. 6), de Charles Bernstein (1987, fig. 7) et de Rosmarie Waldrop (1970, fig. 8), Craig Dworkin (2003: 31-87) a montré comment, aussi concrètes et plastiques soient les silhouettes formées sur la page, le texte ne cesse d'y insister pour ne pas céder la place à l'image. Et si ces analyses vont dans le sens du célèbre «Il n'y a pas de hors-texte» de Derrida (1967 : 227-228), puisqu'elles vérifient que le texte n'a ni commencement ni fin, qu'il n'a ni contexte ni extérieur que l'on puisse lui opposer, qu'il n'est pas clos sur lui-même et qu'en conséquence, il est partout, on pourrait tout aussi bien écrire, en paraphrasant le philosophe : « il n'y a pas de hors-langue » (y compris et même dans la poésie numérique, serait-on tenté d'ajouter). La langue fait toujours retour dans l'image ou dans le médium, même quand ils semblent vouloir l'expulser ; elle n'a de cesse de s'infiltrer partout où elle semble ne plus avoir droit de cité, jusque dans la muette matérialité de l'œuvre. 
Fig. 6. Poèmes de Susan Howe, The Nonconformist's Memorial, New York, New Directions, 1993.
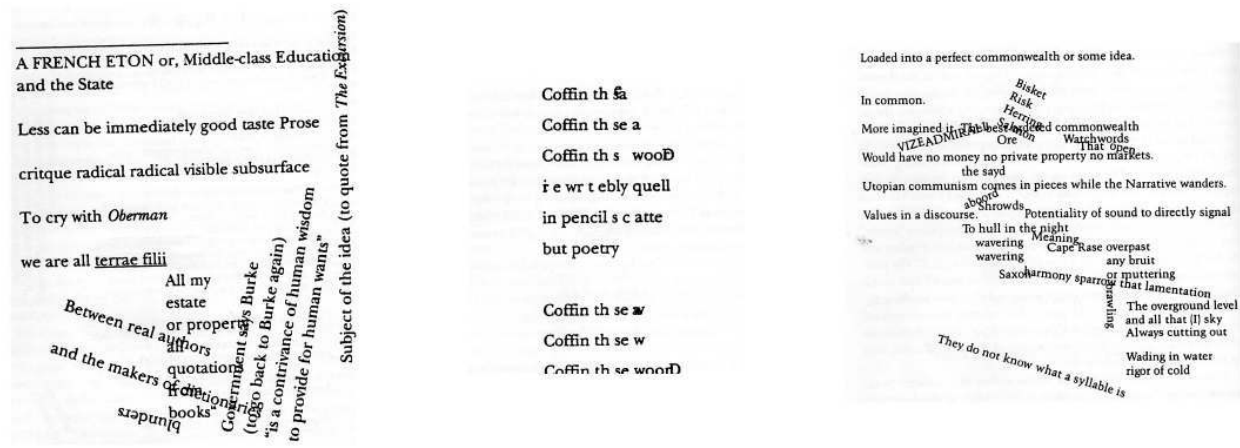

Fig. 7. Extraits de Charles Bernstein, Veil, Madison, Xexoxial, 1987.
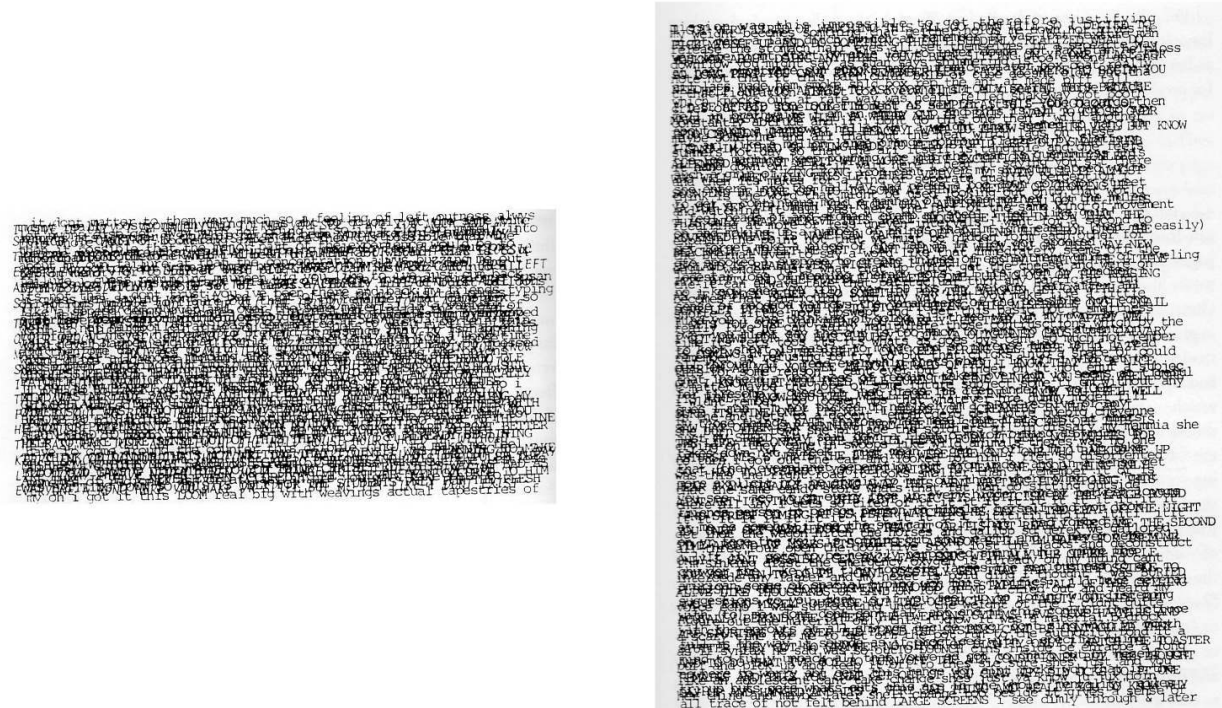
Fig. 8. Rosmarie Waldrop, Camp Printing, Providence, Burning Deck, 1970.
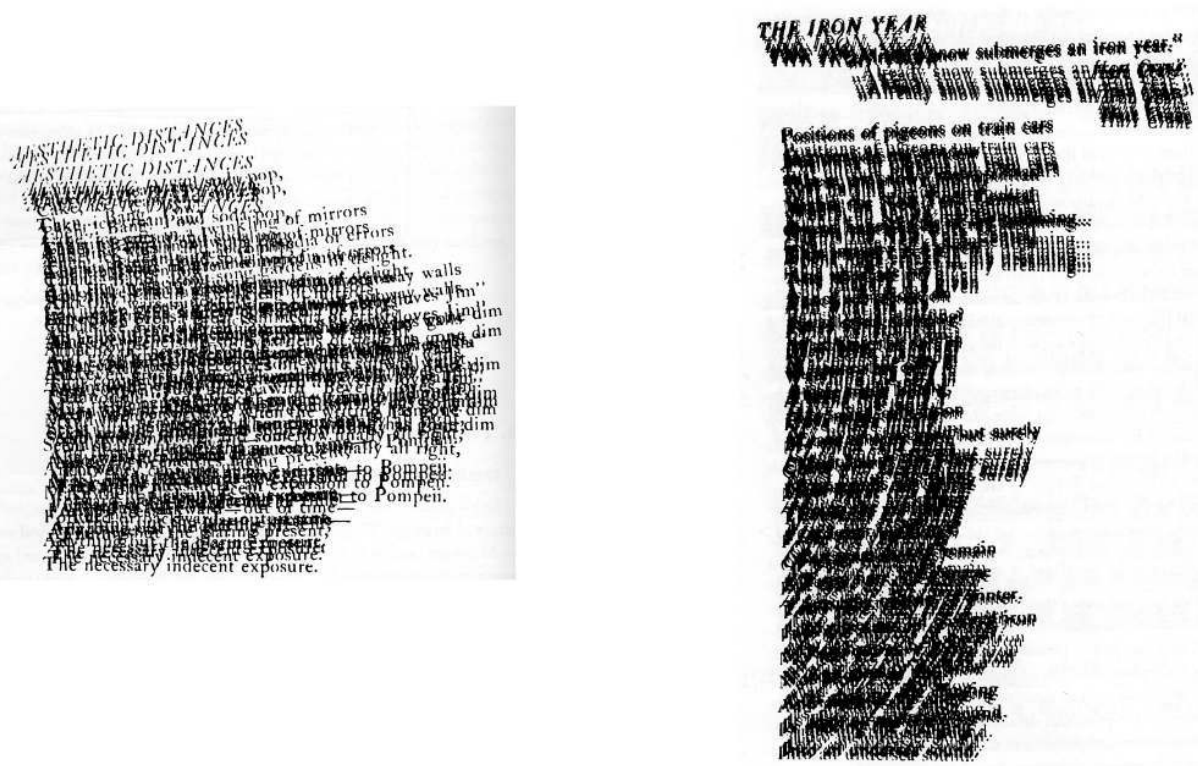

Réciproquement, la poésie numérique suggère que la langue est toujours déjà le support du poème. Cette leçon n'est pas neuve : au début du xx siècle, Ernest Fenollosa ([1918] 1972 : 12-15, 26-34, 45-56) avait intitulé son ouvrage sur l'idéogramme chinois The Chinese Written Character as a Medium for Poetry. Il y défendait l'idée que le système de notation des langues chinoises constituait un outil poétique de choix pour l'Occident, puisqu'il permettait de concaténer en un même caractère les images de réalités étrangères l'une à l'autre, sinon contraires. Lus dans la perspective linguistique et historique ouverte par Fenollosa, les Cantos d'Ezra Pound (1989) nous apprennent qu'il n'y a pas de « révolution du langage poétique » qui ne reprenne d'abord à neuf la question du support de la poésie (Kristeva 1974 : 607-619). Le médium, en somme, détermine ici le patron rythmique dans lequel vient se mouler la langue.

Comment se manifeste cette détermination réciproque du langage et du médium ? Quand Brian Kim Stefans (2000) conçoit le code qui permet d'afficher The Dreamlife of Letters ${ }^{6}$ sur un écran, il l'écrit dans une langue qui n'est pas sa langue maternelle (le langage informatique) mais il conçoit aussi un support à part entière : le code est le médium, certes immatériel, du phénomène graphique et visuel qu'est le poème apparaissant sur un écran. Sur le plan théorique, la poésie numérique propose donc à son lecteur une nouvelle compréhension des rapports entre langage et médium. Or, ces rapports relèvent d'une certaine façon et de manière métaphorique de la traduction: le passage du code à l'affichage du poème met en jeu une traduction intermédiale ou intersémiotique, puisque l'on passe d'un système de signes à un autre (Jakobson 1963 : 78-86). Mais cette opération est interne à la conception du poème, propre à sa nature technique, puisque l'entité « code / poème affiché » n'existe qu'à contenir en elle-même la traduction qui engendre le poème à partir du code. Dans le même ordre d'idée mais de manière différente, le mixte de texte et d'images que met en œuvre Jacques Donguy (2017) dans ses pièces « Pure Data " n'est pas réductible à l'une ou l'autre de ces composantes. Le rapport des typographies entre elles, les effets et les couleurs appliqués au texte font que celui-ci n'est 
jamais simplement le texte, mais il n'est jamais, par ailleurs, plus que le texte. Donguy (2006: 366) parle à ce sujet d'« écriture verbi-voco-visuelle ». Ainsi, la conjonction du texte et de l'image réalise un en-deçà et un au-delà du langage. Les pièces de Jacques Donguy sont tout à la fois moins que du langage, puisque la figuration (faire apparaître des formes) y remplace en partie la symbolisation (coder le réel en signes), et plus que du langage puisque l'image et le mouvement s'ajoutent aux signes et que ceux-ci sont augmentés d'une dimension plastique, graphique et matérielle qui, tout comme chez Alessandro de Francesco, déborde la symbolisation. Le travail sur le support est donc aussi un travail sur le langage ou plutôt sur ce qui, dans ce qui semble hors langage, vient cadrer le langage.

\section{Détours de la traduction}

La poésie numérique nous pousse donc à opter pour une entente élargie des concepts de traduction et de «lecture traductrice », et à les étendre du champ de la langue à celui du médium. Tout se passe en réalité comme si le processus de traduction était inscrit à même le support numérique et déployé dans le passage invisible du code à l'affichage du poème. Ou plus exactement, le processus de traduction est inscrit dans la technique même du poème numérique et rendu nécessaire par cette dernière, puisqu'elle suppose un code et une apparition. Lorsque je lis Der krimgotische Fächer d'Oskar Pastior (1978), je suis confronté à une langue qui, parce qu'elle écartèle les coordonnées usuelles du Hochdeutsch sous un afflux incontrôlé de langues étrangères, de dialectes et d'argots divers, me boute hors du processus de lecture traditionnel pour me faire lire « en traduction » et en « plus d'une langue ». Pastior (1987 : 34-35 ; 1994 : 66-67) a élaboré les poèmes de son recueil sans recourir à un idiome de référence, à partir d'emprunts à plusieurs langues qu'il croise dans un "dialecte de synthèse ». Le jeu des langues, concaténées dans des motsvalises et propulsées par l'énergie de l'écriture dans un espace privé de coordonnées sémantiques stables, est une réponse au concept de Weltliteratur de Goethe (1848: 206). Pastior lui préfère cependant la dissémination babélienne des idiomes, l'espace incertain et sans hiérarchie des dialectes et des minorités. Les poèmes du Krimgotische Fächer sont ainsi à la fois incompréhensibles et ouverts sur un vertigineux infini de significations indécidables et inépuisables. La traduction que je dois effectuer pour lire le texte, et parfois simplement le déchiffrer, se joue entre le texte, l'ensemble des langues qu'il mobilise et ma maîtrise de la langue allemande, qu'il met à mal. Voici à titre d'exemple l'un des poèmes du Krimgotische Fächer.

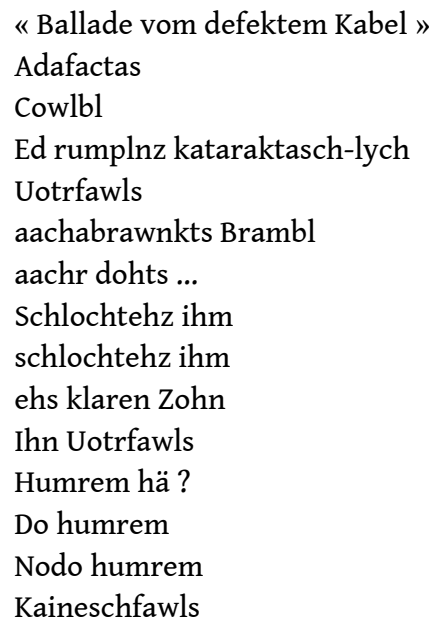




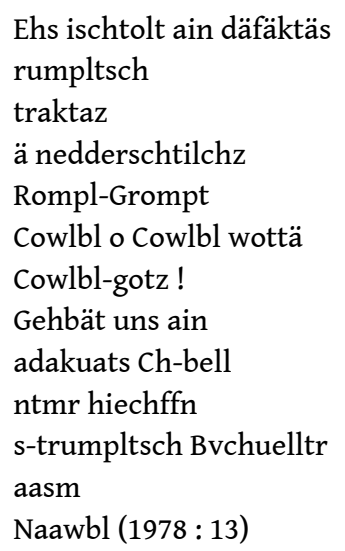

En revanche, lorsque je lis ${ }^{7}$ Jean-Pierre Balpe ou les Lettres dérangées ${ }^{8}$ de Patrick-Henri Burgaud (2005) sur mon ordinateur, c'est la machine qui opère la lecture traductrice à ma place. C'est elle qui, à partir d'un texte donné et d'un code, procède à l'affichage du texte selon les mouvements que j'imprime à la souris. Tout comme dans une traduction linguistique, il y a du jeu et de l'aléatoire ici aussi : le texte qui s'affiche à l'écran n'est pas le simple fruit d'un calcul mais réclame l'intervention d'une subjectivité. Mais alors que la traduction mise en jeu par la poésie textualiste est interlinguistique, la traduction dans la poésie numérique ne correspond à aucune des catégories identifiées par Jakobson (1963). Je propose donc de la nommer "traduction métasémiotique ", puisqu'elle réunit plusieurs systèmes de signes (code, texte, formes visuelles, sons, etc.) et organise leur dialogue par un processus qui s'apparente à la traduction. Du reste, la poésie numérique peut mobiliser le processus de traduction de bien d'autres manières. Ainsi de Poetrica ${ }^{9}$ de Giselle Beiguelman (2003), un dispositif qui affiche, dans l'espace urbain, des textes en polices de caractères non phonétiques (des signes tels que $\mathrm{O}=\diamond$, ou $\bigcirc$ et 0 ). Les typographies utilisées dans Poetrica relèvent à la fois du textuel, de l'illisible et de l'imprononçable. Aussi impliquent-elles un processus avorté de traduction, puisque le lecteur est nécessairement confronté à son désir de compréhension et à l'impossible déchiffrement du texte.

Dissémination babélienne des idiomes pour la poésie textualiste, dissémination des supports pour la poésie élargie : je formulais plus haut l'hypothèse que l'incarnation de la poésie élargie en une multitude de sites avait peut-être disséminé également les interrogations sur le langage elles-mêmes. Cette dissémination expliquerait pourquoi, très souvent, nous sommes déçus devant la poésie numérique, notamment parce que nous ne retrouvons pas, dans l'expérience du poème, l'intensité linguistique à laquelle nous a habitués la poésie qui a marqué l'histoire. Peut-être faut-il simplement poser l'hypothèse que cette dissémination est, dans certains cas, une déperdition tout sauf féconde, comme si elle entraînait également la perte de cette densité, de cette Dichte qui définit la poésie, étymologiquement et historiquement ${ }^{10}$. Nul besoin de le déplorer : c'est la règle de la littérature expérimentale que d'essayer ses formes, quitte à faire l'épreuve de leur caducité rapide et à les abandonner pour passer à d'autres formes (Prigent 2004: 102-106). Dans la poésie élargie, et notamment la poésie numérique, la manipulation du langage cède la place à une manipulation des formes, qu'elles soient sonores, visuelles ou typographiques. Lorsqu'il nous arrive d'être déçus, peut-être le poème n'expérimente-t-il plus sur ce qui nous semble être le cœur de la poésie, à savoir une recherche en acte sur le langage, mais sur ses parerga, c'est-à-dire « ce qui manque en elle [...], vide substantiel, absence déterminable et bordée [...], l'impossibilité d'arrêter la différence en son contour, 
d'arraisonner l'hétérogène (la différance) dans la pose » (Derrida 1978 : 93). Autrement dit, sur tout ce qui déborde les langues par des formes non linguistiques. Est-ce à dire que la poésie numérique n'est plus de la poésie? Plutôt que de répondre par l'affirmative, et risquer de tomber ainsi dans le piège essentialiste, je préférerais répondre que la poésie numérique nous pousse au contraire à abandonner nos réflexes perceptifs: notre déception ne serait alors que l'effet de cet abandon. Comme le montrait déjà Chklovski en 1917 (2008 : 23-25), celui-ci revêt une fonction émancipatrice: nous échappons dans le même temps aux modes de pensée et aux idéologies auxquels la société et l'art ordinaire nous assignent. Et en effet : les avant-gardes textualistes nous poussaient à lire la poésie selon un paradigme venu de Clement Greenberg ([1961] 1989 : 148-153, 171-175, 226-248). D'après le critique d'art américain, la peinture se réduirait, au cours de son histoire, à ses constituants essentiels, la surface et le pigment. Il est facile d'adapter cette conception de l'histoire à la littérature : au cours de son histoire, la poésie se réduirait graduellement à ses constituants fondamentaux, à savoir la langue et les phonèmes. La poésie numérique remet ce paradigme en question en travaillant sur d'autres éléments que le langage. Peutêtre faut-il alors voir dans la permutation et l'animation des signes le moyen spécifique par lequel la poésie numérique retrouve la densité sémantique et textuelle qui définit la poésie. On retrouve en effet ces jeux de permutations dans bien des poèmes numériques, par exemple dans First Screening ${ }^{11}$ de bpNichol (1984), dans Passage de Philippe Bootz (1997, fig. 9) ou dans Code Movie 1 de Giselle Beiguelman (2004). De même que Christian Prigent (1976) ouvre des chemins de lecture alternatifs dans Hacettepe University Bulletin (fig. 10) ou que Baudelaire ([1861] 1975 : 26-27) joue de doubles sens étymologiques dans «La Chevelure » et invite à lire, sous le texte, une constellation de rapports qui n'apparaît pas d'abord dans la syntaxe $d u$ poème, le texte numérique recèle, grâce aux permutations, d'autres textes possibles (si l'on considère que le texte est la configuration de signes affichée sur l'écran à un instant $t$ ) et par conséquent d'autres significations potentielles.

Fig. 9. Extrait de Philippe Bootz, Passage, 1997.

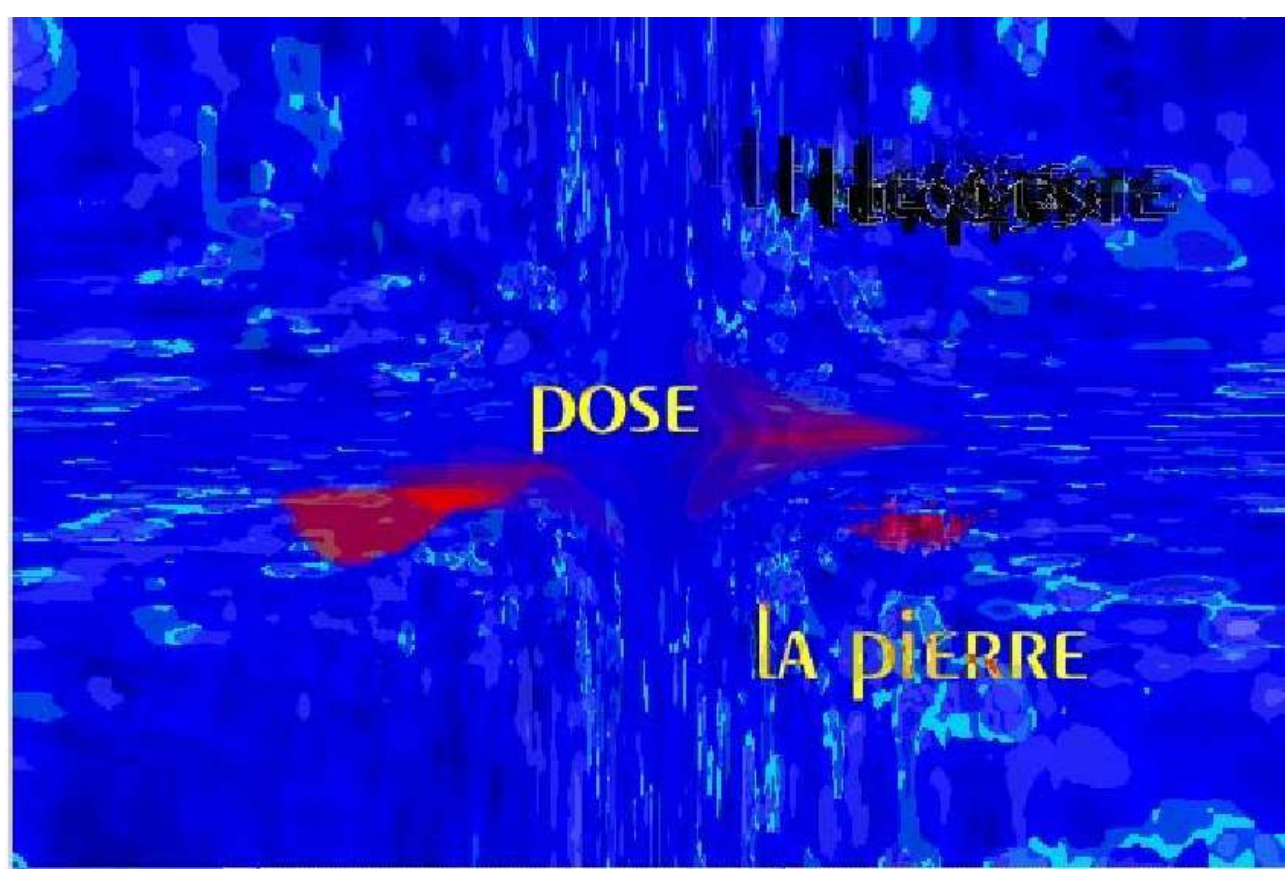

Itinéraires, 2017-3 | 2018 
Fig. 10. Christian Prigent, Hacettepe University Bulletin, Nœuds-les-Mines, Alin Anseeuw, 1976.
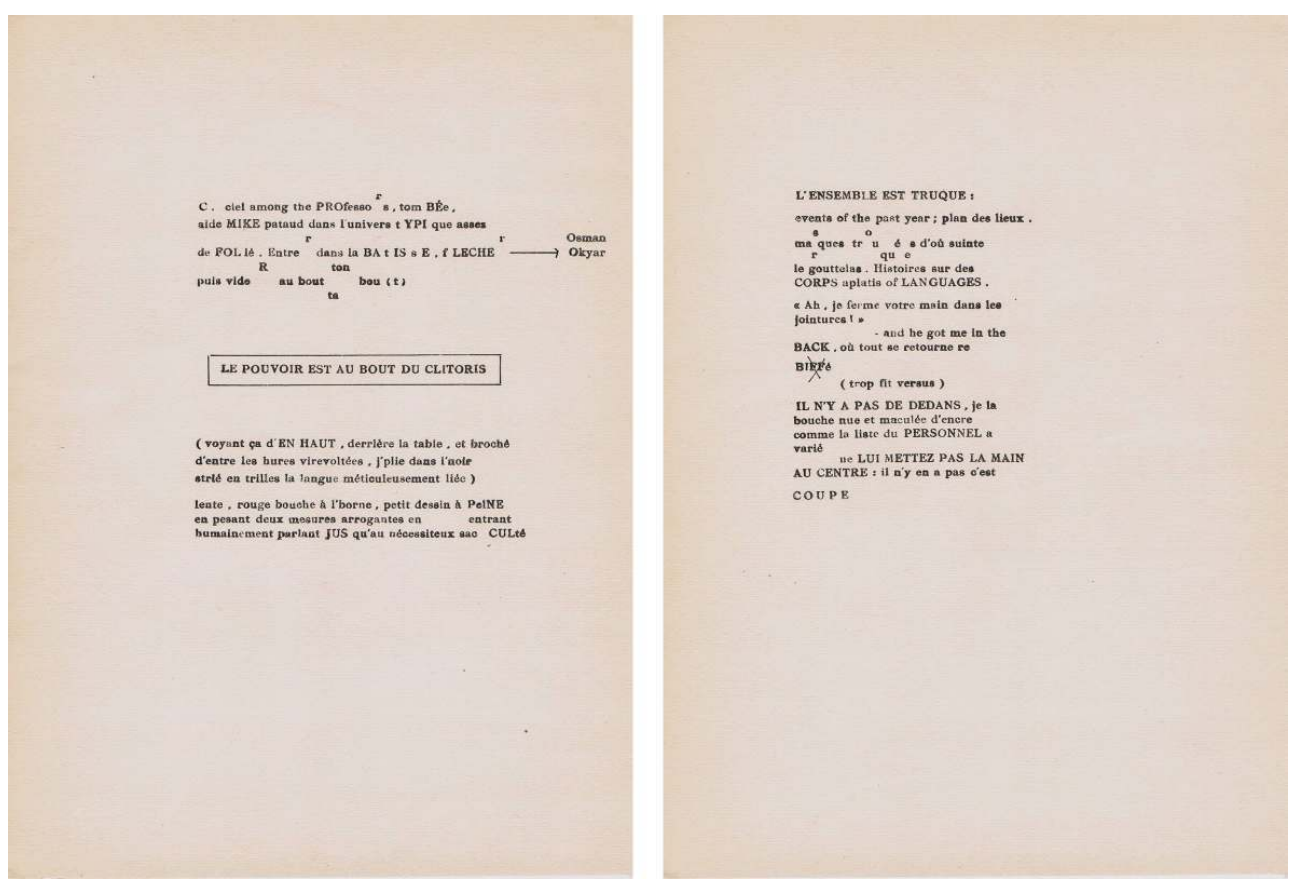

Quel que soit le champ poétique dont on parle, la traduction porte en elle le processus émancipateur qui définit le projet moderne et avant-gardiste. Éric Dayre (2009: 5-7, 19-20) définit ainsi la traduction comme une "écriture dissemblable» à la langue courante et aux représentations ordinaires. Au début de L'Absolu comparé, il écrit :

La traduction est ce qui arrive à la langue. [...] Une traduction, c'est-à-dire l'insistance indéfinie d'une autre écriture face à la langue maternelle, est déstabilisante pour une théorie poétique qui se voudrait fondée sur l'identité de la langue, et sur l'idée selon laquelle le style serait d'abord l'affaire d'une seule langue à la fois.

Dans la poésie textualiste, la dissémination des langues, en multipliant les foyers linguistiques auxquels se rattache le sujet, délivre ce dernier de l'assignation à une origine, une langue et un lieu uniques, et crée un espace à la fois plurilingue et conflictuel. Jacques Derrida (1972 : 379) le décrivait en ces termes :

Tour de Babel où les langues et écritures multiples se heurtent et passent les unes dans les autres, se transforment et s'engendrent depuis leur altérité la plus irréconciliable, la plus affirmée aussi car la pluralité ici n'a pas de fond et n'est pas vécue sans négativité, dans la nostalgie de l'unité perdue.

La confrontation des langues crée un espace propre au texte poétique, espace de significations inépuisables et instables, comme dans le Krimgotische Fächer de Pastior (1978). Dans la poésie élargie, c'est la dissémination des supports qui prend en charge ce processus de « désassignation » et qui produit cet espace d'instabilité. Le code ou le texte numérique est cette autre écriture qui insiste indéfiniment face à nos réflexes de lecture. La poésie élargie est à la page imprimée ce que le texte en « plus d'une langue » est à la langue maternelle. 


\section{Conclusion}

20 L'origine de cet article est un constat simple : le peu de visibilité dont jouit la poésie numérique alors qu'elle a désormais près de soixante ans. Depuis les premières expériences de Theo Lutz, Nanni Balestrini, Jean Baudot (1964), Brion Gysin et Ian Sommerville jusqu'à la dernière livraison de l'Electronic Literature Collection (2016), en passant par la revue Alire dans les années 1990, elle semble demeurer en état indéfiniment expérimental. En fin de compte, c'est un débat daté (même s'il a été pertinent en son temps) qui lui fait de l'ombre, celui de l'affrontement entre littéralisme et lyrisme. L'hypothèse d'une dissémination des supports propre à la poésie numérique, que j'ai développée plus haut, me semble expliquer en partie ce manque de publicité. Non seulement la relativité et la versatilité des supports numériques compliquent l'accès du lecteur à cette littérature ${ }^{12}$, mais elles la rendent invisible en partie. Lorsqu'un support devient obsolète, c'est toute la production qui y est fixée qui devient, d'un seul coup, inaccessible. D'une certaine façon, l'immatérialité de la poésie numérique ne fait pas le poids face aux revues et aux livres imprimés. Par conséquent, les poétiques modernes, fondées sur des questions linguistiques, ont encore la part belle face aux littératures postmodernes a priori fondées sur le support, et donnent même le sentiment d'une opposition entre les premières et les secondes.

21 En écrivant cet article, je me suis tenu en observation à la surface de l'histoire ; il m'a bien semblé que le moderne s'oppose au postmoderne ${ }^{13}$. Non parce que le second chercherait à liquider le premier, comme c'est souvent le cas, mais parce que le premier retarde la venue du second en le dissimulant. Or - et c'est là ce que j'ai tâché de montrer ici -, sous cet effet de surface, il apparait surtout que modernité et postmodernité réciproquement s'anticipent et se prolongent plus qu'elles ne s'affrontent. D'abord parce qu'il n'y a pas de réflexion sur le langage qui ne se fonde sur une réflexion matérialiste consacrée aux supports d'inscription (Kristeva 1974: 607-619); ensuite parce que la réflexion sur le médium porte en puissance une réflexion sur la langue. La traduction et ses mécanismes apparaissent ici comme le trait d'union qui les relie : elle fait passer la langue dans le médium et confond celui-ci avec celle-là. Puisque la traduction engage une conception matérialiste du langage, dans la mesure où elle manifeste en acte qu'il n'existe pas de pensée qui transcenderait les idiomes et que l'on pense toujours dans la matière même de la langue, elle montre comment les réflexions relatives aux «matières textuelles sur supports numériques» (Saemmer 2007: 11-14, 19-25) sont encore des réflexions sur le langage et réciproquement. Entre la continuation du projet moderniste par d'autres moyens et son remplacement par ces mêmes moyens, l'histoire hésite. Elle hésite d'autant plus que, depuis que l'art contemporain est sorti de la «clôture de l'histoire " (Danto [1997] 2000 : 155-176), modernité et postmodernité existent désormais simultanément. Pour Arthur Danto ([1992] 1996 : 291-308), le passage de l'une à l'autre implique la fin du projet moderniste et, avec elle, celle d'une histoire orientée. Pourtant, lorsque l'on observe la production poétique contemporaine, il semble plutôt que la postmodernité n'interdit pas la résurgence du projet moderniste. Cette hésitation mène à une impasse d'autant plus forte que la poésie numérique rejoue parfois le geste esthétique des poétiques expérimentales modernes - la transformation des formes, grosse d'une transformation de l'histoire (Faye 1968) - sans nécessairement en réactiver la signification politique. Or il importe que l'expérimentation continue d'endosser une 
fonction politique, comme dans certains doubles sens des textes de First Screening (1984) où la tautologie apparente tâche en réalité de penser cette fonction.

Plutôt que de voir dans le numérique une porte de sortie ou une nouvelle voie nécessairement salutaire pour la poésie, peut-être faut-il chercher alors des solutions poétiques nouvelles dans les pratiques qui traitent d'un même geste les questions du langage et du support : chez Vincent Broqua (2015 : 135-218) quand, après une série de pages-écrans, il intitule "Traductions (Épreuves du divers)» la dernière partie de Récupérer et qu'il s'y interroge sur la nature de la langue qu'il parle, tout en y intégrant dessins, photographies et notes manuscrites; chez Michaël Batalla (2015: 186-187) lorsqu'il questionne le lien entre la silhouette graphique du texte sur la page et ses emprunts à d'autres idiomes; chez Jean-Pierre Balpe quand il conçoit le générateur linguistique Labilogue avec Maurice Benayoun et Jean-Baptiste Barrière, qu'il réalise Capture pour la Gaité lyrique avec Gregory Chatonsky ou qu'il compose ses technopoèmes, ou encore chez Jérôme Game quand il applique un même principe de découpage au texte écrit ou lu, à la matière sonore qui accompagne ses textes enregistrés (2013) ou aux images qui composent ses vidéopoèmes (2007). Mais plus encore, c'est l'attention donnée à la portée politique ou critique du geste expérimental qui, aujourd'hui comme hier, tient la poésie à bonne distance des impasses de toutes sortes et de la vanité du pur jeu esthétique.

\section{BIBLIOGRAPHIE}

Barthes, Roland, 2002, Euvres complètes, Paris, Seuil.

Bense, Max, 1969, Einführung in die informationstheoretische Ästhetik. Grundlegung und Anwendung in der Texttheorie, Reinbek, Rowohlt.

Bolter, Jay David, 1991, Writing Space. The Computer, Hypertext, and the History of Writing, Hillsdale, Erlbaum.

Bolter, Jay David et Grusin, Richard, 2000, Remediation. Understanding New Media, Cambridge, MA, MIT Press.

Bootz, Philippe, 2006, « De la poésie à la poésie numérique : approche sémiotique », $D O C(K) S$, quatrième série, $n^{\circ}$ 1/2/3/4, p. 378-383, [En ligne], http://www.akenaton-docks.fr/DOCKSdatas_f/collect_f/auteurs_f/B_f/BOOTZ_F/TEXTES_f/delapoesieLasie\%20.htm, consulté le 20 juin 2017.

Cassin, Barbara, 2012, Plus d'une langue, Montrouge, Bayard.

Chklovski, Victor, [1917] 2008, L’Art comme procédé, trad. Régis Gayraud, Paris, Allia.

Collectif, 1958, « Note différentielle sur les Ultra-Lettrismes de Villeglé, Dufrêne et Estivals », -grâmmeS, $\mathrm{n}^{\circ} 2$, p. 7-8.

Cramer, Florian, 2004, « Über Literatur und Digitalcode », dans F. W. Block, C. Heibach, et K. Wenz (dir.), poes1s: Ästhetik digitaler Poesie, Ostfildern-Ruit, Hatje Cantz, p. 263-276. 
Danto, Arthur, [1992] 1996, Après la fin de l'art, trad. Claude Hary-Schaeffer, Paris, Seuil.

Danto, Arthur, [1997] 2000, L'Art contemporain et la Clôture de l'histoire, trad. Claude Hary-Schaeffer, Paris, Seuil.

Dayre, Éric, 2009, L’Absolu comparé. Littérature et traduction, Paris, Hermann.

Derrida, Jacques, 1967, De la grammatologie, Paris, Minuit.

Derrida, Jacques, 1972, La Dissémination, Paris, Seuil.

Derrida, Jacques, 1978, La Vérité en peinture, Paris, Flammarion.

Di Manno, Yves, 1995, La Tribu perdue. Pound vs. Mallarmé, Paris, Java.

Donguy, Jacques, 1985, 1960-1985, une génération. Poésie concrète, poésie sonore, poésie visuelle, Paris, Artefact.

Donguy, Jacques, 2006, « Panorama de la poésie numérique : vers une écriture verbi-vocovisuelle », $\operatorname{DOC}(K) S$, quatrième série, $\mathrm{n}^{\circ} 1 / 2 / 3 / 4$, p. 366-371, [En ligne], http://www.akenatondocks.fr/DOCKS-datas_f/collect_f/auteurs_f/D_f/DONGUY_f/TXT_F/THEORIE.htm, consulté le 20 juin 2017.

Donguy, Jacques, 2007, Poésies expérimentales, zone numérique, 1957-2007, Dijon, Les Presses du Réel. Dworkin, Craig, 2003, Reading the Illegible, Evanston, Northwestern University Press.

Faye, Jean-Pierre, 1968, « Montage, production », Change, $\mathrm{n}^{\circ}$ 1, p. 5-13.

Fenollosa, Ernest, [1918] 1972, Le Caractère écrit chinois, matériau poétique, trad. Ghislain Sartoris, Paris, L'Herne.

Gitelman, Lisa, 2006, Always Already New: Media, History and the Data of Culture, Cambridge, MA, MIT Press.

Gleize, Jean-Marie, 2011, « Opacité critique », dans Collectif, « Toi aussi, tu as des armes ». Poésie \& politique, Paris, La Fabrique, p. 27-44.

Goethe, Johann Wolfgang von, [1848] 1998, Conversations de Goethe avec Eckermann, trad. Jean Chuzeville, Paris, Gallimard.

Greenberg, Clement, [1961] 1989, Art et culture. Essais critiques, trad. Ann Hindry, Paris, Macula. Jakobson, Roman, 1963, Essais de linguistique générale, t. I, trad. Nicolas Ruwet, Paris, Minuit. Kant, Immanuel, [1790] 1993, Critique de la faculté de juger, trad. Alexis Philonenko, Paris, Vrin. Kristeva, Julia, 1974, La Révolution du langage poétique. L'avant-garde à la fin du XIXe siècle, Paris, Seuil.

Lutz, Theo, 1960, « Über ein Programm zur Erzeugung stochastisch-logistischer Texte », Grundlagenstudien aus Kybernetik und Geisteswissenschaft, vol. 1, n 1, p. 11-16.

Maulpoix, Jean-Michel, 2000, Du lyrisme, Paris, José Corti.

Maulpoix, Jean-Michel, 2002, Le Poète perplexe, Paris, José Corti.

McLuhan, Marshall, 1962, The Gutenberg Galaxy. The Making of Typographic Man, Toronto, University of Toronto Press.

Pastior, Oskar, 1987, Jalousien aufgemacht. Ein Lesebuch, Munich, Hanser.

Pastior, Oskar, 1994, Das Unding an sich. Frankfurter Vorlesungen, Francfort-sur-le-Main, Suhrkamp. 
Prigent, Christian, 2004, Ne me faites pas dire ce que je n'écris pas. Entretiens avec Hervé Castanet, Saussines, Cadex.

Prigent, Christian et Gorillot, Bénédicte, 2009, Christian Prigent, quatre temps. Rencontre avec Bénédicte Gorillot, Paris, Argol.

Rose, Heidi M., 2006, « The Poet in the Poem in the Performance: The Relation of Body, Self and Text in ASL Literature ", dans H.-D. L. Bauman, J. L. Nelson et H. M. Rose (dir.), Signing the Body Poetic: Essays on American Sign Language Literature, Berkeley, University of California Press, p. 130-146.

Saemmer, Alexandra et Dufrêne, Bernadette, 2014, « Patrimoines éphémères ", Hybrid, $\mathrm{n}^{\circ}$ 1, [En ligne], http://www.hybrid.univ-paris8.fr/lodel/index.php?id=174, consulté le 20 juin 2017.

Saemmer, Alexandra, 2007, Matières textuelles sur support numérique, Saint-Étienne, Publications de l'Université de Saint-Étienne.

\section{Corpus}

Andre, Carl, 2014, Poems, Zurich, JRP/Ringier.

Apollinaire, Guillaume, [1918] 1956, Calligrammes, dans Euvres poétiques, Paris, Gallimard.

Batalla, Michaël, 2015, Poésie possible, Caen, Nous.

Baudelaire, Charles, [1861] 1975, Les Fleurs du mal, dans CEuvres complètes, t. I, Paris, Gallimard.

Baudot, Jean, 1964, La Machine à écrire, Montréal, Éditions du Jour.

Beiguelman, Giselle, 2003, Poetrica, http://www.desvirtual.com/projects/poetrica/, consulté le 20 juin 2017.

Beiguelman, Giselle, 2004, Code Movie 1, http://collection.eliterature.org/1/works/ beiguelman__code_movie_1/, consulté le 20 juin 2017.

Bernstein, Charles, 1987, Veil, Madison, Xexoxial.

Bootz, Philippe, 1997, Passage, http://www.labo-mim.org/site/index.php?passage2, consulté le 20 juin 2017.

bpNichol, 1984, First Screening : Computer Poems, http://vispo.com/bp/videoversion.htm, consulté le 20 juin 2017.

Broqua, Vincent, 2015, Récupérer, Paris, Les petits matins.

Burgaud, Patrick-Henri, 2005, Jean-Pierre Balpe ou les Lettres dérangées, http://

collection.eliterature.org/1/works/burgaud__jean-pierre_balpe_ou_les_lettres_derangees.html, consulté le 20 juin 2017.

Burroughs, William S., 1961, The Soft Machine, Paris, Olympia Press.

De Francesco, Alessandro, 2013, Augmented Writing, Rome, La Camera Verde.

De Francesco, Alessandro, 2016, La Vision à distance, trad. Caroline Zekri et al., Paris, Mix.

Demarcq, Jacques, 1988, La Danse du dos, Xonrupt-Longemer, Æncrages.

Demarcq, Jacques, 2008, Les Zozios, Caen, Nous.

Donguy, Jacques, 2017, Pd-extended 1. Poésie numérique en Pure Data, Paris, Les Presses du réel. 
Electronic Literature Organization, 2016, Electronic Literature Collection, http://

collection.eliterature.org/3/, consulté le 20 juin 2017.

Game, Jérôme, 2007, Ceci n'est pas une légende ipe pe ce, Marseille, Incidences.

Game, Jérôme, 2013, DQ/HK, Bordeaux, L'Attente.

Gomringer, Eugen, 1995, Vom Rand nach Innen. Die Konstellationen 1951-1995, Vienne, Splitter.

Heidsieck, Bernard, 2008, Vaduz, Limoges, Al Dante.

Howe, Susan, 1993, The Nonconformist's Memorial, New York, New Directions.

Lutz, Theo, 1959, «Stochastische Texte », augenblick, quatrième année, nº 1, p. 3-9.

Pastior, Oskar, 1978, Der krimgotische Fächer. Lieder und Balladen, Erlangen, Renner.

Pound, Ezra, 1989, The Cantos, New York, New Directions.

Prigent, Christian, 1975, L'Main. Organon I, Paris, L'énergumène.

Prigent, Christian, 1976, Hacettepe University Bulletin, Nœuds-les-Mines, Alin Anseeuw.

Prigent, Christian, 1977, Power/Powder. Organon II, Paris, Bourgois.

Prigent, Christian, 1978, Euf-glotte. Organon III, Paris, Bourgois.

Prigent, Christian, 1982, Voilà les sexes, Paris, Luneau Ascot.

Prigent, Christian, 1984, « La voix-de-l'écrit (lectures publiques) », TXT, nº 17, p. 33-38.

Prigent, Christian, 1996, Rien qui porte un nom. Quelques peintres, Saussines, Cadex.

Prigent, Christian, 1997, L'Écriture, ça crispe le mou, Meuvy-le-Roy, Alfil.

Stefans, Brian Kim, 2000, The Dreamlife of Letters, http://collection.eliterature.org/1/works/ stefans_-the_dreamlife_of_letters.html, consulté le 20 juin 2017.

Waldrop, Rosmarie, 1970, Camp Printing, Providence, Burning Deck.

\section{NOTES}

1. https://vimeo.com/23577896 (cette vidéo est l'enregistrement d'une performance datée du 17 mai 1996 ; Christian Prigent y lit deux textes. Le premier, "Litanie », est tiré du livre Voilà les sexes (Prigent, 1982); la lecture de Liste des langues que je parle commence à 4:30) ; http:// www.lyrikline.org/en/poems/liste-des-langues-que-je-parle-11998-.WTaw-9SLTDc.

2. https://www.youtube.com/watch?v=CUPuZzPaFJM.

3. http://www.desvirtual.com/bio/.

4. http://www.alessandrodefrancesco.net/TEP_RE.html.

5. Les images qui composent la fig. 5 sont issues du site http://frenchpoetryand.wordpress.com/ et son reproduites avec l'aimable autorisation de Nina Parish et d'Emma Wagstaff, ainsi que celle d'Alessandro de Francesco.

6. https://www.youtube.com/watch?v=ZSnq0nMAQQc.

7. Le sens du verbe « lire» est ici multiple, puisqu'il renvoie autant à la lecture du texte qu'à l'exécution du programme sur l'ordinateur.

8. http://nt2.uqam.ca/fr/repertoire/jean-pierre-balpe-ou-les-lettres-derangees-0.

9. http://www.medienkunstnetz.de/works/poetrica/video/1/. 
10. En allemand, "poésie » se dit «Dichtung», tandis que "Dichte » désigne la densité. Kant souligne l'étymologie commune à ces deux mots lorsqu'il écrit que la poésie conduit à « une plénitude de pensées » (Kant [1790] $1993: 230)$.

11. https://www.youtube.com/watch?v=rEdUSQ7WCSM.

12. La multiplicité des supports implique des questions très simples mais pourtant redoutables pour qui cherche à lire de la poésie numérique. Où chercher? Comment savoir ce qui est disponible sur tel ou tel support? Avec quel matériel et quels logiciels accéder à ces productions ? L'absence de politique patrimoniale dédiée à la littérature numérique n'aide bien sûr pas à régler ces questions. Quant aux livres et aux revues, le dépôt légal et le catalogue de la Bibliothèque nationale de France sont de précieux outils. En dernier recours, le lecteur curieux peut toujours se rendre à la BnF pour trouver des textes qui ont disparu de la circulation.

13. On pourrait ainsi affilier aux poétiques modernes des auteurs comme Jacques Demarcq, Christian Prigent ou Jean-Pierre Verheggen et associer aux poétiques post-modernes des auteurs comme Philippe Bootz, Jean-Pierre Balpe ou Jacques Donguy.

\section{RÉSUMÉS}

Cet article étudie les modes d'expérimentations des avant-gardes poétiques textualistes et de la poésie numérique. Il montre que l'expérimentation sur le support s'est substituée à l'expérimentation sur les langues mais qu'en réalité, ces deux enjeux sont profondément liés. L'article fait ainsi l'hypothèse que les poétiques postmodernes prolongent le projet moderne plus qu'elles n'y mettent fin, et tâche de mieux comprendre la logique de l'évolution de la poésie expérimentale au $\mathrm{xx}^{\mathrm{e}}$ siècle, en dépit de l'éclatement de ses formes.

Focusing on modes of experimentation in textualist avant-garde as well as digital poetry, this paper argues that experimentation on the medium has replaced experimentation on languages, though both modes remain deeply connected. Post-modern poetics, it shows, renews modernist poetry rather than puts an end to it. Thus it attempts to provide a better understanding of the evolution of experimental poetry during the twentieth century, beyond its innumerable forms.

\section{INDEX}

Keywords : medium, language, translation, avant-garde, multilingualism, intermediality, experimentation, poetry, digital poetry, poetry in the expanded field

Mots-clés : médium, support, langue, traduction, plurilinguisme, intermédialité, expérimentation, avant-garde, poésie, poésie numérique, poésie élargie

\section{AUTEUR}

\section{MATHIAS KUSNIERZ}

CERILAC - Université Paris-Diderot 